\title{
Previewer for Multi-Scale Object Detector
}

\author{
Zhihang $\mathrm{Fu}^{*}$ \\ Zhejiang University \\ Alibaba Group \\ zhihangf@zju.edu.cn \\ Chen Shen* \\ Zhejiang University \\ Alibaba Group \\ zjushenchen@gmail.com
}

\author{
Zhongming Jin \\ Alibaba Group \\ zhongming.jinzm@alibaba-inc.com
}

\author{
Rongxin Jiang $\dagger$ \\ Zhejiang University Embedded \\ System Engineering Research Center, \\ Ministry of Education of China \\ Xian-Sheng Hua $\uparrow$ \\ Alibaba Group \\ xiansheng.hxs@alibaba-inc.com
}

\author{
Guo-Jun Qi \\ University of Central Florida \\ guojun.qi@ucf.edu
}

\author{
Yaowu Chen \\ Zhejiang Provincial Key Laboratory \\ for Network Multimedia Technologies
}

(1)
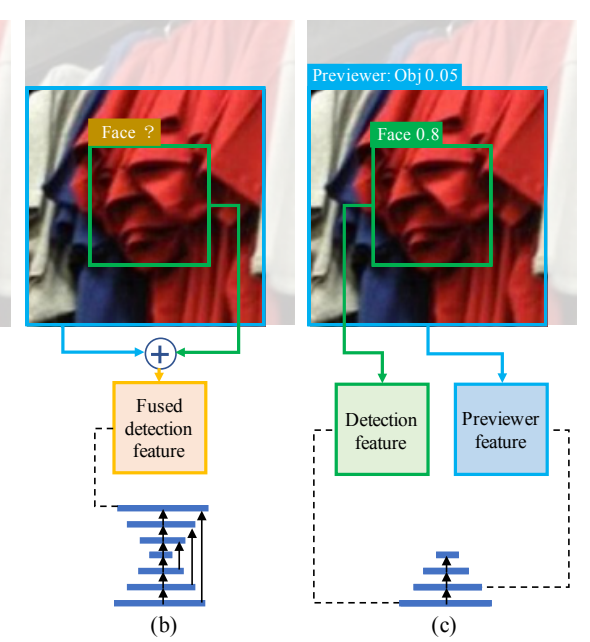

Figure 1: (a) A false positive occurs on the wrinkles of Tshirts due to limited size of receptive field. (b) Top-down structure method fuses features for better feature expressions. (c) A light-weight previewer proposed in this paper addresses the problem by previewing broader.

\section{INTRODUCTION}

Convolutional Neural Networks (CNN) based methods [4, 9, 15, $25,32,35]$ have significantly improved the performances of object detection task in recent years. They are capable of extracting strong semantic features as to boost object detection. However, the performance usually declines when dealing with the images containing objects of a wide range of sizes. Feature pyramids built upon multi-scales of images $[10,26]$ are conventional solutions to this issue, but it is computationally extensive and time consuming, making the methods impractical for real applications. Thus, many popular CNN-based detectors turn to leverage different feature layers for detecting objects at different scales, among which are SSD [25], MS-CNN [3] and Hierarchical Gated Deep Network (HGDN) [27]. The large resolution of low-level features enables small size sliding-windows for detecting small objects. 
Unfortunately, useful contextual information is missed due to the weakness and small receptive field sizes of low-level features. As shown in $[6,20,27,28]$, contextual information indeed plays a great role in object detection, especially for small objects. The lack of contextual information leads to unsatisfactory performance of multi-scale detectors on detecting small objects. Moreover, the number of small object priors is large (accounts for over $66 \%$ of the whole prior boxes) due to the large resolution of low-level feature layers. Thus most of false positives tend to lie on small priors in multi-scale detectors. An intuitive example is shown in Figure 1(a).

Top-down architecture is proposed in [23] to enhance semantics in low-level features and many other methods such as [12, 21, 24, 36] share the same insight through feature fusion among different receptive fields. Top-down feature fusion mechanism could improve object detection results, but it is time consuming due to the heavy structure, which is shown in Figure 1(b).

The goal of this paper is to explicitly and independently predict all object prior boxes twice with different receptive field sizes by leveraging the CNN's feature hierarchy. Specifically, we first preview the objectness probabilities for the potential regression regions of prior boxes on the deeper feature layers with sufficiently larger receptive fields that involve enough contextual information. Then we further classify and relocate them on the layers/receptive fields in conventional settings. To this end, we first verify that most false positives occur on low-level feature layers, and then propose a novel previewer block that could be easily embedded into all multi-scale detectors such as SSD [25] and MS-CNN [3]. We also formulate a new matching strategy to select positive/negative training examples for the previewer block. The results show that impressive improvements can be reached after previewing prior boxes. We also demonstrate that even the detectors with feature fusion mechanisms such as RFBNet [24] would perform better through adding the proposed previewer block.

Our contributions in this paper are summarized below.

- First, we prove that independent predictions from different feature layers on the same region is beneficial for a detector, even for those who already have feature fusion mechanisms.

- Second, we propose a previewer block to show that explicitly constraining features at different layers can provide additional benefits. Previewer block performs as well as heavy top-down structure, but much faster than it.

- Finally, experiments are conducted on PASCAL VOC [11] and KITTI [13] pedestrian benchmark. We show that previewer block greatly improves all three multi-scale detectors: SSD, RFBNet and MS-CNN.

\section{RELATED WORK}

In this section, we briefly review the recent $\mathrm{CNN}$-based algorithms on object detection, then pay special attention to the relationship between receptive field and prior box size, and conventional designs of objectness score.

\subsection{Object Detection}

R-CNN [15] first introduces CNN into object detection task. In RCNN, proposals generated by Selective Search [40] are cropped as patches from the original images. These patches are sent into a CNN such as AlexNet [22] and ZFNet [42], then final classification and localization results are computed from the fixed-length vectors extracted from CNN. Based on R-CNN, SPP net [17], Fast R-CNN [14] and Faster R-CNN [32] are proposed successively. With the remarkable performance, Faster R-CNN becomes one of the most popular baselines. It proposes region proposal network (RPN) and prior box which is inspired by MultiBox [39]. The idea of prior box promotes the development of detection and almost all the subsequent algorithms [3, 16, 23, 25, 30] inherit this design.

Along with Faster R-CNN, two-stage detection algorithms continue to grow and eventually form a large family. MS-CNN [3] is one variant focusing on detecting objects in KITTI datasets [13]. RPN in MS-CNN generates proposals at multiple output layers. Subsequently, FPN [23] also chooses multi-scale output design for proposals with top-down architectures. Many latest algorithms, such as Mask R-CNN [16], inherit FPN design to cope with the challenges of the wide range of object scales.

On the other hand, SSD [25] and YOLOv2 [30] are two typical single shot detectors in recent years. They both use prior boxes and directly output detection results without time consuming RoI pooling operation [32]. SSD uses multiple output layers to ensure the fit matching between receptive field sizes and object scales. RFBNet [24] proposes Receptive Field (RF) block module which fuses different features generated by atrous convolutions $[5,19]$ to enhances the discrimination and robustness of features. DSSD [12] involves top-down architecture to enhance high-resolution semantically weak features. According to [33], RFBNet and DSSD can be regard as two ways of feature fusion by introducing a stronger object classifier and a stronger feature extractor respectively. Both methods improve the performance of detection.

\subsection{Receptive Field vs. Prior Box Size}

MultiBox [39] and Faster R-CNN [32] first introduce prior box into CNN-based detection algorithms. In Faster R-CNN, all prior boxes (named as "anchors" in that paper) are tiled in the same layer (conv5_3 in VGG [37]). The receptive field size of conv5_3 is 196 and the priors have 3 scales $\left(128^{2}, 256^{2}, 512^{2}\right)$ and 3 aspect ratios $(1: 1,1: 2,2: 1)$. It is not quite reasonable that receptive field size is much smaller than the size of many prior boxes.

In contrast, SSD [25] allocates priors at different scales on different feature layers to fitly match the sizes of corresponding receptive field. It is worth noting that the size of priors is always set to be smaller than receptive field size. So SSD is even better than Faster R-CNN using the same backbone VGG16 without ROI pooling for feature alignment. Furthermore, Faster R-CNN overtakes SSD when they both use ResNet101 [18] as backbone [12, 16], which is deeper with large enough receptive field size in RPN layer for Faster R$\mathrm{CNN}$. It confirms the view from the opposite side that receptive field should be larger than the size of prior boxes to involve more contextual information.

However, the larger receptive field size is not always the better for object detection. In KITTI pedestrian detection [13], for example, the object size is pretty small (about $20 \times 50$ ) while the image size is large $(1250 \times 375)$. Semantic information of small objects would fade with the down-sampling of feature maps and it will lead to accuracy decline for both classification and regression. So 


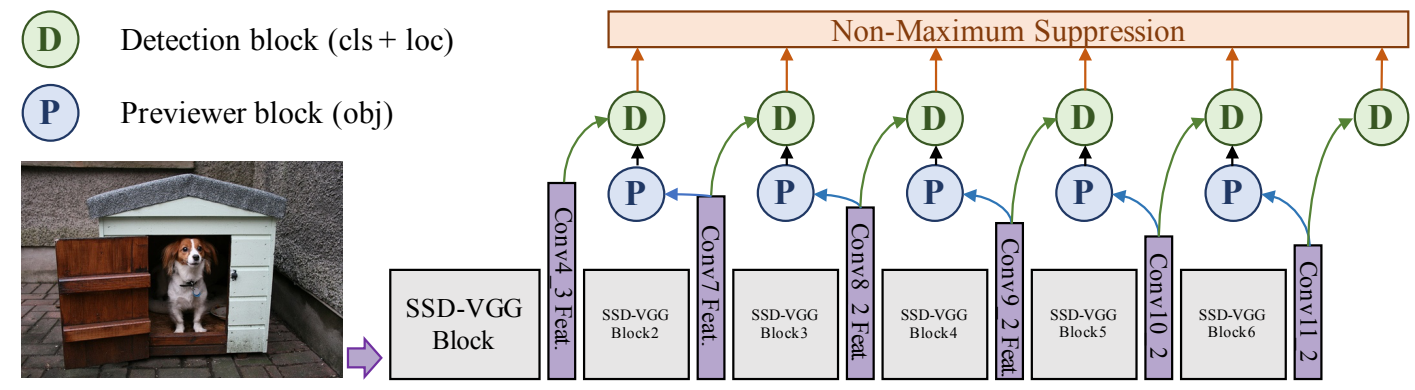

Figure 2: Overview of the previewer for a multi-scale detector. Previewer blocks first preview the potential regression region of each prior box, using the adjacent deeper layers to predict the objectness probabilities. Then finer localizations and classspecific classifications are conducted based on the previewing results.

MS-CNN [3] is proposed to match prior boxes with proper receptive fields in different layers, and Qi [27] presents a hierarchical gated network structure to selectively fuse multi-scale information based on the image context across different pixel locations.

\subsection{Objectness Score}

As affirmed by the early works $[1,2,40]$ that objectness is a generic concept and a universal objectness detector can be learned from data, the RPN in Faster R-CNN based method outputs objectness score for each prior box which measures membership to a set of object classes vs. background. These two stage methods first "preview" where there exists objects by objectness score in RPN, and then make more accurate predictions of what kind of the objects are (classification) and where they are (regression).

SSD [25] does not have objectness score predictions and directly outputs the classification scores of all prior boxes. In contrast, YOLO $[29,30]$ predicts objectness scores and classification scores simultaneously. The objectness score in YOLO reflects how confident the model is that the box contains an object and how accurate the bounding box is that the model predicts. However, the objectness scores and classification confidences are predicted from the same feature layer in YOLO. It means that YOLO cannot involve more contextual information without changing backbone net when predicting the objectness score.

Recently a novel research focusing on real-time object detection, namely R-FCN-3000 [38], also involves objectness score prediction. The method decouples objectness detection and classification of the detected objects to fix computational requirements for localization when the number of classes increases. But classification and objectness detection are both predicted from conv5 layer (ResNet50) and share the same features and receptive field in R-FCN-3000.

\section{PREVIEWER NETWORKS}

Inspired by RPN in Faster R-CNN which picks a few high-quality proposals first from tens of thousands anchors and YOLO that predicts whether a region contains objects, we propose novel previewer blocks to preselect regions with high confidences containing objects by involving enough contextual information. The detector then classifies and relocates the prior boxes in these regions. Figure 2 illustrates the proposed network architecture and all steps are finished in a single shot fashion.

\subsection{Previewer Block}

The goal of proposed block is to preview the regions, which are larger than corresponding prior boxes, whether contain objects or not. It is a binary classification task and can be also formulated as predicting objectness scores.

The critical differences between our objectness prediction and those mentioned in section 2.3 can be summarized as follows: 1 ) We decouple previewer block and detection task by using deeper feature layers which have sufficiently larger receptive field sizes to preview whether the regions truly have objects. The type of false positives shown in Figure 1 can be effectively suppressed when seeing broader. 2) What we preview are regions rather than the specific prior boxes, which are contained within the former. It does not make sense to predict whether a prior box contains objects because the final predictions would have offsets or size changes based on the prior boxes. What's more, classification has been already done on prior boxes and it would leads to homogenization if previewer block also detects objectness on the same areas. In stead, we preview the whole large region where a prior box might be regressed. The objectness prediction on this kind of region should be more stable even though the final prediction bounds are shifted from the original prior boxes.

The details of proposed previewer block are illustrated in Figure 3. Our design principle is to improve the original detector with the light-weight modifications. First, we prove in section 4.1 that most false positives usually occur on low-level, large-resolution feature layers for multi-scale detectors. Thus the previewer blocks are implemented in the framework to enhance detection, especially for the large-resolution feature layers, namely conv4_3 and conv7 layer in SSD-VGG16. Second, different with the priors that could have more than one box at the same sliding position of a feature layer, we just preview one fixed region which is large enough to involve efficient contextual information as well as contain all priors at the same sliding window position. This region predicts one objectness score for all priors contained in it (4-6 prior boxes per previewer region), as shown in Figure 3(a). Third, because the manifold of interest is low-dimensional (only 1-channel output for predicting objectness of each region), we draw on the idea of Inverted Residuals in MobileNetv2 [34] by using shortcuts directly between the bottlenecks. Specifically, the intermediate features in the block are thick (256 channels) while the input and output 


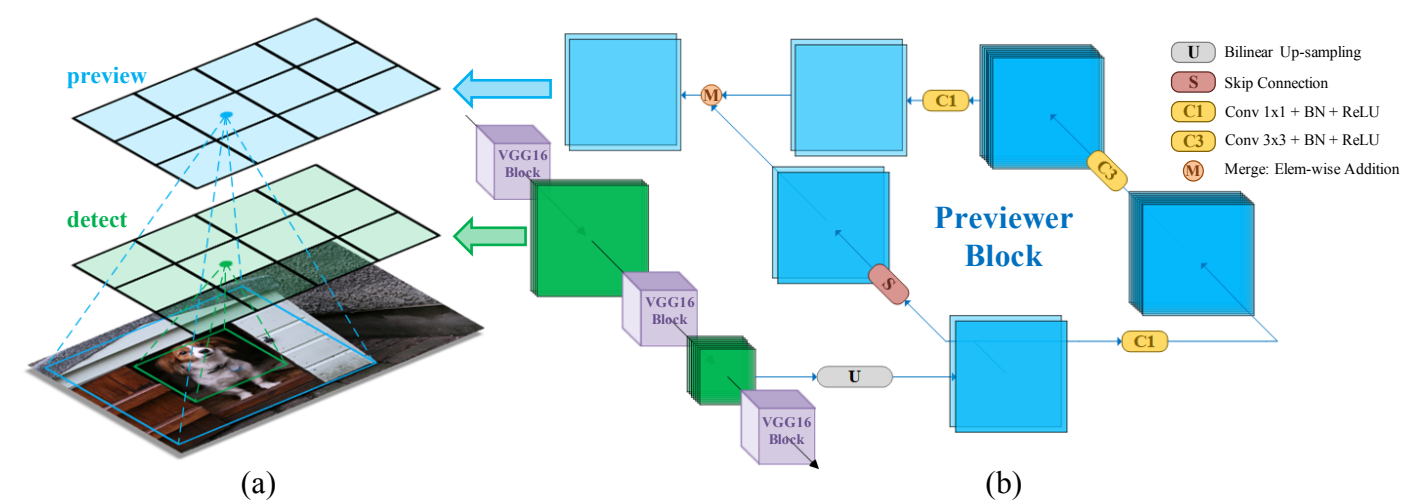

Figure 3: (a) The relationship between previewing and detecting. (b) Details of previewer block. We first up-sample the features by bilinear interpolation, and then use a light-weight block which is similar to inverted residuals: output channel number is small (64) meanwhile the intermediate channel number is large (256) to enhance the block.

features are thin (64 channels) to shrink the dimensions. Figure 3(b) tells the details.

\subsection{Matching Strategy}

As mentioned in section 3.1, we use larger receptive fileds to preview the region.The goal of previewer block is to check whether a certain region contains objects or not, so it is no longer reasonable to assign a positive or negative label to a prior box by measuring IoU (Intersection of Union) ratio with ground truth boxes. We introduce the metric IoG (Intersection of Ground-truth) ratio to formulate the containment relations between the previewer region and groundtruth bounding boxes.

$$
I o G_{i}=\max _{j=1,2, \ldots, N} \frac{S_{\text {previewer_region }} \cap S_{\text {ground }_{-} \text {trut }_{j}}}{S_{\text {ground-truth }_{j}}}
$$

where $N$ is the number of ground-truth objects. An object is completely contained by the previewer region when IoG $=1.0$, and we assign a positive label to this region. A previewer region will get a negative label if IoG $<0.8$. What's more, the label of a larger region, which for example is in conv7 and contains an object, is set to be ignored (neither positive nor negative during training) when that object is already contained in smaller previewer region in conv4_3.

After the matching step, most of these previewer regions are negatives and we involve hard negative example mining similar with the operations in SSD [25]. We keep the ratio between negatives and positives to be 1:1 and find that this leads to a more stable training and better results compared with other ratios.

\subsection{Loss Function and Output Mode}

The proposed method has three outputs: objectness scores Prob(obj) from previewer block, conditional classification scores $\operatorname{Prob}(\mathrm{cls} \mid \mathrm{obj})$ and the location offsets based on prior boxes.

$\operatorname{Prob}(\mathrm{cls} \mid o b j)$ is conditioned on $\operatorname{Prob}(o b j)$ and the final prediction result is $\operatorname{Prob}(\mathrm{cls})=\operatorname{Prob}(\mathrm{cls} \mid o b j) \times \operatorname{Prob}(o b j)$. We use cross-entropy loss function to optimize the outputs of $\operatorname{Prob}(\mathrm{cls} \mid o b j)$ and use binary cross-entropy loss (BCE loss) on Prob(obj). The localization loss is a Smooth L1 loss [14] between the predicted box and the ground-truth box. We regress to offsets for the center point coordinates, width and height of the prior box, which is similar to Faster R-CNN [32] and SSD [25].

In summarization, we define a multi-task loss as $L=L_{(c l s \mid o b j)}+$ $L_{o b j}+L_{r e g}$. The loss on previewer block $L_{o b j}$ is average BCE loss and the ground-truth label is assigned following the strategy described in section 3.2.

We also introduce two output modes for our detectors. The normal one is multiplication of the conditional class probabilities and the objectness probabilities: $\operatorname{Prob}(c l s)=\operatorname{Prob}(c l s \mid o b j) \times \operatorname{Prob}(o b j)$, which is called soft mode in this paper. The hard output mode is that we first pick high-quality proposals by dropping those regions with the low objectness scores under a threshold, 0.1 for example. In this mode the preview block is a bit like RPN in Faster R-CNN: they both pick proposals from the massive priors or anchors. We experimentally states that the soft output mode has a better and more stable performance, meanwhile the hard output mode also improves the performance and it could even speedup the inference time with filtering out plenty of predictions before Non-Maximum Suppression (NMS).

\section{EXPERIMENTS}

In this section we first perform preliminary analysis to prove there exists significant room for improvement on suppressing small-scale false positives. Adequate ablation experiments are conducted on PASCAL VOC 2007 dataset. Then we implement the previewer block on SSD and RFBNet successively and state steady improvements on PASCAL VOL dataset. Furthermore, we implement the proposed block on two-stage detector MS-CNN to show its generic compatibilities. With previewer blocks, better results are conducted on KITTI pedestrian benchmark.

\subsection{Preliminary Analysis}

We first dig into the performance of existing multi-scale detector SSD which is trained with PASCAL VOC [11] 2007 trainval set. The training strategy fully confirms to the description in its paper [25] and we get the final mAP $70.3 \%$ after 60k iterations, which is 2.3-point higher than the results $(68.0 \%)$ in its paper [25]. This baseline result is reasonable and the increments come from random 


\begin{tabular}{lcccccc}
\hline Threshold & 0.5 & 0.3 & 0.23 & 0.2 & 0.1 & 0.01 \\
TP number & 7481 & 8179 & 8468 & 8614 & 9195 & 10457 \\
FN number & 4551 & 3853 & 3564 & 3418 & 2837 & 1575 \\
FP number & 1405 & 2615 & 3510 & 4068 & 8517 & 109965 \\
\hline Recall & $62.2 \%$ & $68.0 \%$ & $70.4 \%$ & $71.6 \%$ & $76.4 \%$ & $86.9 \%$ \\
Precision & $84.2 \%$ & $75.8 \%$ & $70.7 \%$ & $67.9 \%$ & $51.9 \%$ & $8.7 \%$ \\
\hline
\end{tabular}

Table 1: Statistics on True Positive (TP), False Negative (FN) and False Positive(FP) under different confidence thresholds.

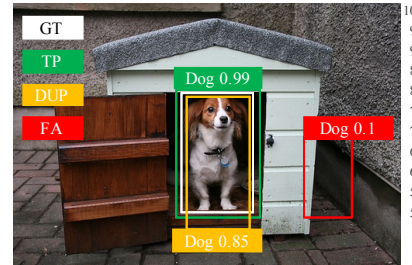

(a)

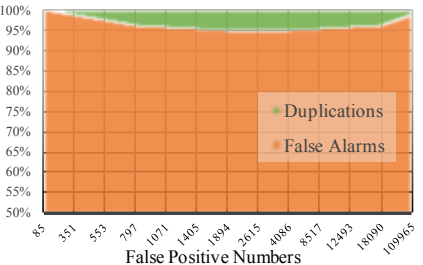

(b)
Figure 4: (a) Illustration of two kinds of false positives: duplications and false alarms. (b) Statistics on duplications and false alarms. False alarm is always the absolute majority.

expansion augmentation mentioned in slides of $\mathrm{SSD}^{1}$. Based on this baseline model, we experimentally affirm that:

False positives occupies a considerable proportion of all failure cases. As shown in Table 1, we evaluate baseline model on VOC 2007 test set and list the number of True Positive (TP), False Negative (FN) and False Positive (FP) under different classification confidence thresholds. There are thousands of FPs even when the threshold is high (0.5). And FP further grows rapidly and overtakes FN when the threshold is about 0.23 . Thus there should be significant room for improvement of detection by suppressing false positives.

False alarm is the main form of false positives. There are two kinds of cases in FP: duplications and false alarms. A prediction is called a duplication box when the IoU, with an already-predicted object, is higher than 0.5 . The others are called false alarms because these predictions have small IoU values with all object bounds and usually locate in background areas. Figure 4(a) illustrates these two cases respectively. We count the proportion of them in Figure 4(b). The results show that false alarm is always the absolute majority (over 95\%) with the increment in the number of false positives. It is reasonable that most duplications are suppressed during the NMS process. The proposed previewer block, aiming at reducing false alarm cases by involving more contextual information, is just motivated by this fact.

Most false positives occur on low-level large-resolution feature layers. We classify false positives according to different sizes and illustrate the distributions in Figure 5. As we pointed in section 1, the number of small-size priors is much larger than that of bigger ones. In SSD, for example, $38 \times 38 \times 4=5776$ prior boxes lie on conv4_3 feature map, which accounts for over $66 \%$ of the total. Another reason is that the receptive field size is limited in

\footnotetext{
${ }^{1}$ http://www.cs.unc.edu/ wliu/papers/ssd_eccv2016_slide.pdf
}

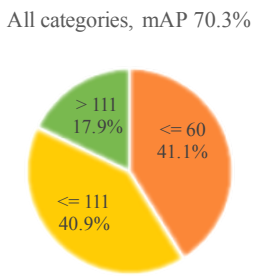

(a)

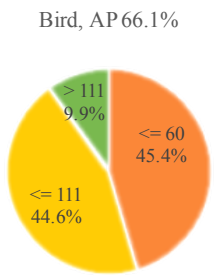

(b)

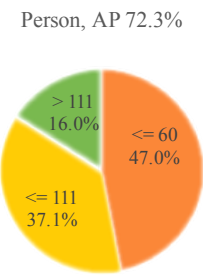

(c)
Figure 5: Statistics on FP of SSD baseline, which is classified by size. The threshold 60 and 111 are the upper bound of prior box size on conv4_3 and conv7 respectively.

\begin{tabular}{lcccccc}
\hline Previewer region size & 60 & 80 & 90 & 100 & 110 & 130 \\
mAP & 71.8 & 72.5 & 72.8 & $\mathbf{7 3 . 1}$ & 72.6 & 72.5 \\
\hline Gains & 1.5 & 2.4 & 2.5 & $\mathbf{2 . 8}$ & 2.3 & 2.2 \\
\hline
\end{tabular}

Table 2: Theoretical gains when previewing the prior boxes on conv4_3 by using ground-truth of the previewer block.

\begin{tabular}{lcccccc}
\hline \multirow{2}{*}{ Model } & \multicolumn{2}{c}{ Previewer loss } & OHNM & Preview & \multicolumn{2}{c}{ mAP on } \\
& cls & obj & ratio & on & Hard & Soft \\
\hline$A$ & $\checkmark$ & & $1: 3$ & region-wise & - & 71.1 \\
$B$ & $\checkmark$ & $\checkmark$ & $1: 3$ & region-wise & - & 71.1 \\
$C$ & & $\checkmark$ & $1: 3$ & region-wise & - & 71.3 \\
\hline$D$ & & $\checkmark$ & NA & region-wise & 70.2 & 70.4 \\
$E$ & & $\checkmark$ & $1: 10$ & region-wise & 71.0 & 71.3 \\
$F$ & & $\checkmark$ & $1: 1$ & region-wise & $\mathbf{7 1 . 1}$ & $\mathbf{7 1 . 6}$ \\
\hline$G$ & & $\checkmark$ & $1: 1$ & prior-wise & 70.1 & 69.9 \\
\hline
\end{tabular}

Table 3: Ablations for previewer block. We train the models on VOC 2007 train set and test on 2007 test set.

low-level features and false alarms are more likely to occur due to lack of contextual information, which is the critical problem that this paper focuses on.

Based on the preliminary conclusions above, we affirm that the performance of multi-scale detectors such as SSD can be further improved through suppressing the large number of false alarms. Without implementing previewer block, we first verify the rationality of our previewer region ideas by applying the ground-truth of previewer block, which is computed with the strategy described in section 3.2, on the baseline SSD model. We explore a wide range of previewer region sizes for the small scale prior boxes in conv4_3 and Table 2 shows the gains in detail. The results indicate that the size of previewer region should be set to about 100 for a better performance and we set it to 104, which is the maximum length of prior boxes in the next (conv7) layer. It is worth noting that the gains in Table 2 could be seen as the theoretical optimal performance when implementing previewer block on SSD, and the truly performance is about 1-point lower shown in section 4.2. Similar experiments are conducted on the rest detection layers and we set the previewer region size to $192,250,320$ and 420 respectively. 


\begin{tabular}{|c|c|c|c|}
\hline \multirow{2}{*}{ Model } & \multicolumn{2}{|c|}{ Embed previewer block into the ___ layer: } & \multirow{2}{*}{ mAP } \\
\hline & same deeper & deeper deeper & \\
\hline$H$ & $\checkmark$ & & 70.7 \\
\hline$I$ & $\checkmark$ & & 71.6 \\
\hline K & & $\checkmark$ & 71.0 \\
\hline
\end{tabular}

Table 4: Different positions for previewer block. "deeper deeper" means we put the previewer block into the position 2 layers deeper than corresponding detection head.

\subsection{Ablation on PASCAL VOC}

We perform ablations on PASCAL VOC to analysis our previewer block. The baseline model (mAP $70.3 \%$ on VOC 2007 test set) is SSD trained on VOC 2007 trainval set, which is described in section 4.1. Ablation results are shown in Table 3 - 5. Details are discussed in the following.

Class-Specific vs. Class-Agnostic. We show 3 different output formats for the proposed previewer block in Table 3. The previewer in model $A$ is class-specific and it outputs 21-channel results representing the probabilities of 20 categories and background in VOC dataset. Thus in model $A$, the outputs of previewer block and classification layer are the same and they are both trained with crossentropy loss. Model $B$ first generates class-specific predictions and then outputs the objectness score (class-agnostic) at each previewer region. These two outputs are constrained at the same time during the training process. Model $C$ directly outputs the class-agnostic objectness scores which are constrained by $\mathrm{BCE}$ loss.

The results of model $A, B$ and $C$ in Table 3 indicate that classagnostic output is preferred on the previewer block. Our intention is only introducing a larger previewer region to check if it contains objects as classifying the category would increase the difficulty of this problem. As a result, we set the outputs of the previewer block as class-agnostic in the following experiments.

Region-wise $v s$. Prior-wise. We report that previewing on the potential regression region of prior boxes (region-wise) is better than previewing on each specific prior box area (prior-wise). In Table 3, model $F$ is the proposed region-wise previewer block. Take conv4_3 layer as an example, the the shape of previewer regions is $104 \times 104,4$ prior boxes contained in the region, as shown in Figure 3.1. In contrast, the previewer region in model $G$ is kept the same with its prior box, and the matching strategy is also the same with that of prior boxes. The results show that region-wise prediction surpasses prior-wise with a large margin. Prediction on prior-wise region is not stable because the final box bound is shifted from the original prior box.

Same Layer $v s$. Deeper Layer. Table 4 shows the reason why we choose the deeper layer for previewer blocks. Model $H$ embeds the previewer block into the same layer with classification head (the block is embedded into conv4_3 to preview the priors on conv4_3, for example.). It is the conventional design used in [30,38]. Model $I$ is the proposed previewer version, and we also try to embed the previewer block into the position 2 layers deeper in model $K$. With the results we remark that one layer deeper is more proper for a previewer block.

Online Hard Negative Mining. We encounter the similar problem with classification sub-task in detection during training the

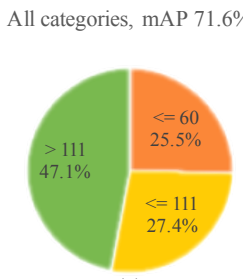

(a)

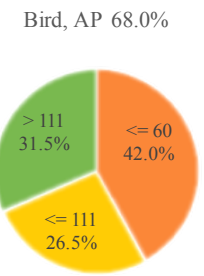

(b)

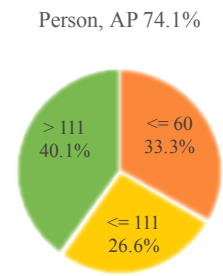

(c)
Figure 6: Statistics on FP of SSD-Previewer. The proportions of small-size FPs drop drastically, compared with Figure 5.

\begin{tabular}{cccc}
\hline \multicolumn{5}{c}{ Preview the priors on the layer: } & mAP \\
conv4_3 & conv7 & conv8_2 & rest detection layers \\
\hline & & & 70.3 \\
$\checkmark$ & & & 71.3 \\
$\checkmark$ & $\checkmark$ & $\checkmark$ & 71.6 \\
$\checkmark$ & $\checkmark$ & $\checkmark$ & 71.0 \\
$\checkmark$ & $\checkmark$ & $\checkmark$ & 71.0 \\
\hline
\end{tabular}

Table 5: Ablations for previewer block. The best results are achieved when the first two detection layers are previewed.

previewer blocks. The dominated negative examples would lead to significant data imbalance and few improvements are achieved in model $D$. We introduce online hard negative mining strategy described in [25] and explore different ratios of positive and negative samples. The results of model $C, E$ and $F$ in Table 3 suggest that $1: 1$ is proper for the previewer block training. However, it also shows that the previewer block is not sensitive to the fluctuation of OHNM ratio. The objectness task in the previewer block is simple but stable during training.

Hard Output $v s$. Soft Output. As described in section 3.3, we introduce two types of previewer outputs during inference time. In hard output mode, the outputs of previewer blocks are first binarized to generate binary masks and final results are obtained by discarding those priors with the mask value 0 . Briefly we treat the previewer block as a first-step filter in this mode, similar to RPN in Faster R-CNN. In soft output mode, we treat the classification predictions as conditional probabilities based on the objectness probabilities generated by the previewer blocks. So we multiply two probabilities to get the final classification results.

The results in Table 3 show that soft output is always better than the hard output. There is one more drawback of hard output: the optimal threshold value for binarizing is not easy to choose and we find that the value is related to OHNM ratios. However, the hard output mode has its merit. It discards plenty of predictions before NMS during inference time, which would reduce NMS time especially when the test image has a high resolution (means there are more priors in total during detecting objects).

Multi-Scale Previewer Blocks. We progressively implement previewer blocks into the framework and compare the results in Table 5. The top layer conv11_2 is not previewed as it already has the largest receptive fields to involve enough contextual information. And the other lower layers are previewed by their adjacent deeper layers. The results in Table 5 show that the more layers for 


\begin{tabular}{lcccc}
\hline Method & Train & Backbone & mAP & FPS \\
\hline Faster R-CNN [18] & $07+12$ & ResNet-101 & 76.4 & 2.4 \\
R-FCN [8] & $07+12$ & ResNet-101 & 80.5 & 6.3 \\
YOLO 448 [29] & $07+12$ & Darknet & 63.4 & 45 \\
YOLOv2 544 [30] & $07+12$ & Darknet-19 & 78.6 & 40 \\
SSD 300 [25] & $07+12$ & VGG-16 & 77.5 & $\mathbf{4 6}$ \\
DSSD 321 [12] & $07+12$ & ResNet-101 & 78.6 & 9.5 \\
RFBNet 300 [24] & $07+12$ & VGG-16 & 80.5 & 43 \\
\hline SSD-Previewer 300 & $07+12$ & VGG-16 & 78.6 & 45 \\
RFBNet-Previewer 300 & $07+12$ & VGG-16 & $\mathbf{8 1 . 2}$ & 43 \\
\hline
\end{tabular}

Table 6: Single-model results on VOC 2007 test set (trained on VOC $2007+2012$ trainval).

previewing is not always the better. The performance is immediately improved when the previewer block is implemented for prior boxes on conv4_3 layer. The reason is that the detection features in conv4_3 are weak in semantic expression and also limited by their small receptive field sizes. Most false positive cases occurs in this layer as shown in the preliminary analysis. With the proposed previewer block, false positives are suppressed due to the deeper and better features and more contextual information. The deep features already have strong semantic information and large receptive fields. Involving larger receptive fields to preview the priors on these layer not only is of little significance, but also makes the task difficult by introducing extra outputs from different layers. Thus we implement 2 previewer blocks for priors on conv4_3 and conv7.

With the proposed previewer block, the distribution of false positives drastically changes from Figure 5 to Figure 6 . The proportion of small-size false positives accounts for a significant reduction. In Figure 5 and Figure 6, we show the FP distributions of bird, person and overall categories. The false positives on conv4_3, on which the the prior sizes are smaller than 60 , and conv7, on which the sizes are smaller than 111 , are successfully suppressed due to previewing and proportion of both is reduced by more than 10 points.

\subsection{PASCAL VOC 2007}

For a fair comparison with other superior detection methods on VOC 2007 test set, we trained our model (SSD-Previewer 300, RFBNetPreviewer 300) using VOC trainval set 2007 and 2012. The classification and regression tasks remain the same as in [25] and we implement previewer blocks to form a objectness recognition task, learned by BCE loss. We use the $10^{-3}$ learning rate for the first $80 \mathrm{k}$ iterations and then continue training for $20 \mathrm{k}$ iterations with $10^{-4}$ and $10^{-5}$. Other training configurations such as batch size, momentum and weight decay remain the same as in SSD. Table 6 shows the results in details.

Top-down structure is not the only way to advance the detection performance. Many recent works [12,21, 23, 36] state that top-down structure facilitates the extraction of better feature at all scales. The result of DSSD [12] in Table 6 confirms this perspective and it outperforms SSD 300 by 1.1 points $(78.6 \% \mathrm{mAP})$ with more effective backbone and top-down structure. However, this kind of feature fusion is inefficient compared with RFBNet, which achieves $80.5 \% \mathrm{mAP}$ with a much higher speed 43 FPS.

\begin{tabular}{lccc}
\hline \multirow{2}{*}{ Method } & \multicolumn{3}{c}{ AP on Pedestrians } \\
& Easy & Moderate & Hard \\
\hline MS-CNN $1280 \times 384[3]$ & 73.7 & 68.4 & 60.7 \\
\hline SSD 300 & 63.9 & 50.1 & 47.5 \\
SSD $1250 \times 375$ & 72.8 & 64.8 & 56.6 \\
SSD $1250 \times 375+$ Previewer & 73.6 & 66.5 & 57.9
\end{tabular}

Table 7: Results of different SSD versions on KITTI pedestrian validation set.

\begin{tabular}{lccc}
\hline \multirow{2}{*}{ MS-CNN Model } & \multicolumn{3}{c}{ AP on Pedestrians } \\
& Easy & Moderate & Hard \\
\hline (a) h384-2x [3] & 76.0 & 69.5 & 61.6 \\
(b) h576-2x [3] & 77.7 & 72.5 & 64.4 \\
(c) h768-2x [3] & 76.3 & 72.7 & 64.3 \\
\hline (d) h384-2x+Previewer & 77.2 & 70.5 & 62.3 \\
(d) h576-2x+Previewer & 78.6 & 73.3 & 65.3 \\
(d) h768-2x+Previewer & $\mathbf{7 8 . 6}$ & $\mathbf{7 3 . 8}$ & $\mathbf{6 5 . 6}$ \\
\hline
\end{tabular}

Table 8: Results of MS-CNN-Previewer on KITTI pedestrian benchmark. All three versions have improved by embedding previewer block.

The previewer block contributes steady improvements for all multi-scale detectors. We implement the light-weight previewer blocks into SSD and get over 1 point improvements in Table $6(78.6 \%$ vs.77.5\%). It proves that previewing regions with larger receptive fields helps a multi-scale detector. Furthermore, RFBNetPreviewer also outperforms the original RFBNet by about 1 point and achieves $81.2 \% \mathrm{mAP}$. It is a fairly remarkable performance for a detector using VGG-16 as a backbone.

Independent predictions with different receptive fields on the same region are necessary even for the detector which already has feature fusion operations (RFBNet). As described in section 2, RFBNet and DSSD both fuse features to pursue better feature expressions. However, the previewer block further predicts the objectness for each region independently with larger receptive field and the results of RFBNet-Previewer 300 prove that it could even advance a detector which already has strong semantic features by feature fusion.

The previewer block is a light-weight head and hardly affects the real-time effects. We embed only 2 previewer blocks into SSD and RFBNet and the proposed block is light-weight compared with top-down structure and the detection head in RFBNet. Results in Table 6 show SSD-Previewer 300 achieves the same performance with DSSD 321 but about 5 times faster.

We also visualize the predictions of objectness and classifications on the same images in Figure 7. It clearly shows why previewer block can suppress false positives and improve the final performance.

\subsection{KITTI Pedestrian Detection}

We experiment on KITTI pedestrian benchmark [13] in this section. KITTI dataset has three levels of evaluation: easy, moderate and hard. The key difficulty of KITTI pedestrian detection task is that a large number of pedestrians are in small size (height $<40$ pixels) and occluded. One of the common failure cases is that false alarms 

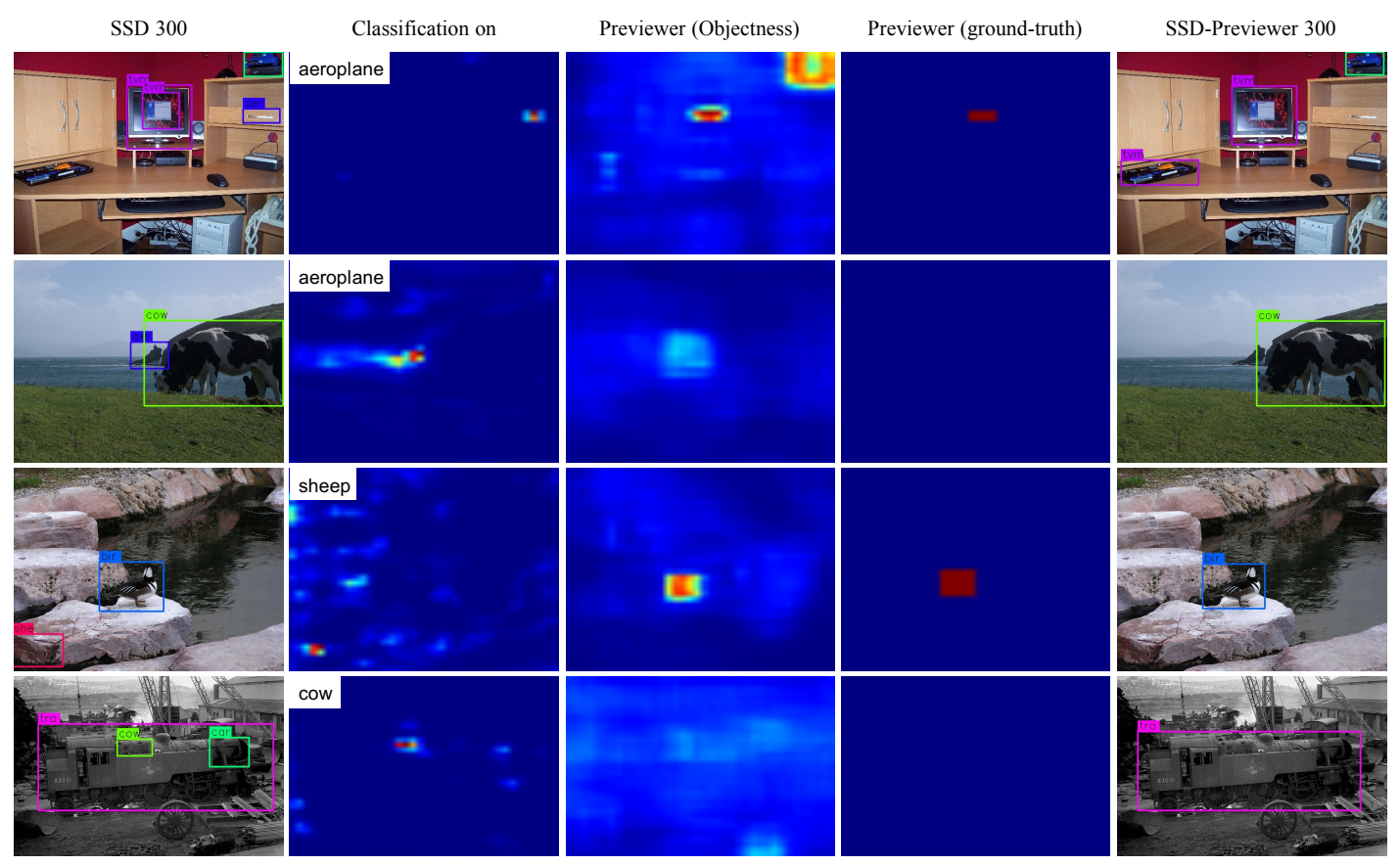

Figure 7: Examples of object detection results. A score threshold of 0.1 is used to draw these bounding boxes. The first column shows results of original SSD. The second column shows the classification scores of each priors on layer conv4_3: blue means low confidence and red means high confidence, and the category of the results is written on the left-top corner of each heat map. Similarly, the third column shows the heat maps of objectness scores for each regression region correspondingly. The objectness confidences on those small-size FP regions are low (blue), which would suppress the final prediction results and reduce $\mathrm{FP}$.

on small-size human-like regions. We report that the proposed previewer block could handle this false alarm related problem by previewing the region with a larger receptive field.

Since the ground-truth annotation of the KITTI testing set is not publicly available, we follow the strategy in [7, 41], splitting the train-val set into training and validation sets to conduct analyses about different methods. The training set contains 3682 images and the validation set contains 3799 images. All comparative experiments below are conducted on training/validation set.

SSD vs. MS-CNN. The shape of KITTI images are extremely wide with the resolution of $1250 \times 375$ and most pedestrians are in small sizes (height $<40$ ). SSD with the conventional configurations perform not well on KITTI because the images are resized to $300 \times$ 300 for training and plenty of fat prior boxes (aspect ratio 1:2 and 1:3) are redundant for pedestrian detection. For a fair comparison with other methods designed specifically for KITTI, we modify the input size to $1250 \times 375$ of SSD, discard the fat priors and optimize the data augmentation strategy to keep the original aspect ratio of images unchanged (named as SSD $1250 \times 375$ in Table 7). And we follow $[3,31]$ to set batch size to 4 during training. We then implement the previewer block in this specialized SSD. The results show that the specialized SSD dramatically improves the performance and the previewer block again increases the average precision. But the MS-CNN is still slightly better as the priors are well assigned according to receptive fields, given the same VGG16 backbone.
Previewer Block in MS-CNN. To prove the previewer block is a generic operation for multi-scale detectors, we implement the previewer block in MS-CNN: the previewer block is trained with the multi-scale RPN and final prediction scores are the product of objectness scores and classification scores. We follow the training strategy in MS-CNN paper [3]. Table 8 reports the results. All three methods have better average precisions when involving previewer blocks.

\section{CONCLUSIONS}

This paper introduces a light-weight previewer block which previews objectness probability for the potential regression region of each prior box in a multi-scale detector. A key insight of our model is independent predictions from different feature layers on the same region is beneficial for detection. The previewer block is simple but generic, and can be easily implemented into multi-scale detectors. Experiments show that the proposed light-weight previewer contributes steady improvements for multi-scale detectors, such as SSD, RFBNet and MS-CNN.

\section{ACKNOWLEDGMENTS}

This paper is partially supported by the Fundamental Research Funds for the Central Universities and NSF under award \#1704309. 


\section{REFERENCES}

[1] Bogdan Alexe, Thomas Deselaers, and Vittorio Ferrari. 2012. Measuring the objectness of image windows. TPAMI 34, 11 (2012), 2189-2202.

[2] Pablo Arbelaez, Michael Maire, Charless Fowlkes, and Jitendra Malik. 2011. Contour detection and hierarchical image segmentation. TPAMI 33, 5 (2011), 898-916.

[3] Zhaowei Cai, Quanfu Fan, Rogerio Feris, and Nuno Vasconcelos. 2016. A Unified Multi-scale Deep Convolutional Neural Network for Fast Object Detection. In ECCV.

[4] Yuanzhouhan Cao, Chunhua Shen, and Heng Tao Shen. 2017. Exploiting depth from single monocular images for object detection and semantic segmentation. IEEE TIP 26, 2 (2017), 836-846.

[5] Liang-Chieh Chen, George Papandreou, Iasonas Kokkinos, Kevin Murphy, and Alan L Yuille. 2018. Deeplab: Semantic image segmentation with deep convolutional nets, atrous convolution, and fully connected crfs. TPAMI 40, 4 (2018), 834-848.

[6] Xinlei Chen and Abhinav Gupta. 2017. Spatial memory for context reasoning in object detection. In ICCV. IEEE, 2980-2988.

[7] Xiaozhi Chen, Kaustav Kundu, Yukun Zhu, Andrew G Berneshawi, Huimin Ma, Sanja Fidler, and Raquel Urtasun. 2015. 3d object proposals for accurate object class detection. In NIPS. 424-432.

[8] Jifeng Dai, Yi Li, Kaiming He, and Jian Sun. 2016. R-FCN: Object Detection via Region-based Fully Convolutional Networks. In NIPS.

[9] Jifeng Dai, Haozhi Qi, Yuwen Xiong, Yi Li, Guodong Zhang, Han Hu, and Yichen Wei. 2017. Deformable convolutional networks. CoRR, abs/1703.06211 1, 2 (2017), 3.

[10] Navneet Dalal and Bill Triggs. 2005. Histograms of oriented gradients for human detection. In CVPR, Vol. 1. IEEE, 886-893.

[11] M. Everingham, L. Van Gool, C. K. I. Williams, J. Winn, and A. Zisserman. 2010 The Pascal Visual Object Classes (VOC) Challenge. IfCV 88, 2 (June 2010), 303338.

[12] Cheng-Yang Fu, Wei Liu, Ananth Ranga, Ambrish Tyagi, and Alexander C Berg. 2017. DSSD: Deconvolutional single shot detector. arXiv preprint arXiv:1701.06659 (2017).

[13] Andreas Geiger, Philip Lenz, and Raquel Urtasun. 2012. Are we ready for Autonomous Driving? The KITTI Vision Benchmark Suite. In CVPR.

[14] Ross Girshick. 2015. Fast R-CNN. In ICCV.

[15] Ross Girshick, Jeff Donahue, Trevor Darrell, and Jitendra Malik. 2014. Rich feature hierarchies for accurate object detection and semantic segmentation. In CVPR.

[16] Kaiming He, Georgia Gkioxari, Piotr Dollár, and Ross Girshick. 2017. Mask r-cnn. In $I C C V$.

[17] Kaiming He, Xiangyu Zhang, Shaoqing Ren, and Jian Sun. 2014. Spatial pyramid pooling in deep convolutional networks for visual recognition. In ECCV. Springer, 346-361.

[18] Kaiming He, Xiangyu Zhang, Shaoqing Ren, and Jian Sun. 2016. Deep residual learning for image recognition. In CVPR. 770-778.

[19] Matthias Holschneider, Richard Kronland-Martinet, Jean Morlet, and $\mathrm{Ph}$ Tchamitchian. 1990. A real-time algorithm for signal analysis with the help of the wavelet transform. In Wavelets. Springer, 286-297.

[20] Peiyun Hu and Deva Ramanan. 2017. Finding tiny faces. In CVPR. IEEE, 15221530.

[21] Tao Kong, Fuchun Sun, Anbang Yao, Huaping Liu, Ming Lu, and Yurong Chen 2017. RON: Reverse connection with objectness prior networks for object detection. In CVPR, Vol. 1. 2.
[22] Alex Krizhevsky, Ilya Sutskever, and Geoffrey E Hinton. 2012. Imagenet classification with deep convolutional neural networks. In NIPS. 1097-1105.

[23] Tsung-Yi Lin, Piotr Dollár, Ross Girshick, Kaiming He, Bharath Hariharan, and Serge Belongie. 2017. Feature pyramid networks for object detection. In CVPR, Vol. 1. 4.

[24] Songtao Liu, Di Huang, and Yunhong Wang. 2017. Receptive Field Block Net for Accurate and Fast Object Detection. arXiv preprint arXiv:1711.07767 (2017).

[25] Wei Liu, Dragomir Anguelov, Dumitru Erhan, Christian Szegedy, Scott Reed, Cheng-Yang Fu, and Alexander C Berg. 2016. SSD: Single Shot Multibox Detector. In ECCV. Springer, 21-37.

[26] David G Lowe. 2004. Distinctive image features from scale-invariant keypoints. IfCV 60, 2 (2004), 91-110.

[27] Guo-Jun Qi. 2016. Hierarchically gated deep networks for semantic segmentation. In CVPR. 2267-2275.

[28] Guo-Jun Qi, Xian-Sheng Hua, Yong Rui, Jinhui Tang, and Hong-Jiang Zhang. 2010. Image classification with kernelized spatial-context. IEEE TMM 12, 4 (2010), 278-287.

[29] Joseph Redmon, Santosh Divvala, Ross Girshick, and Ali Farhadi. 2016. You only look once: Unified, real-time object detection. In CVPR. 779-788.

[30] J. Redmon and A. Farhadi. 2017. YOLO9000: Better, Faster, Stronger. In CVPR. 6517-6525. DOI : http://dx.doi.org/10.1109/CVPR.2017.690

[31] Jimmy Ren, Xiaohao Chen, Jianbo Liu, Wenxiu Sun, Jiahao Pang, Qiong Yan, Yu-Wing Tai, and Li Xu. 2017. Accurate Single Stage Detector Using Recurrent Yu-Wing Tai, and Li Xu. 2017.
Rolling Convolution. In CVPR.

[32] Shaoqing Ren, Kaiming He, Ross Girshick, and Jian Sun. 2015. Faster r-cnn: Towards real-time object detection with region proposal networks. In Advances in NIPS. 91-99.

[33] Shaoqing Ren, Kaiming He, Ross Girshick, Xiangyu Zhang, and Jian Sun. 2017. Object detection networks on convolutional feature maps. TPAMI 39, 7 (2017), 1476-1481.

[34] Mark Sandler, Andrew Howard, Menglong Zhu, Andrey Zhmoginov, and LiangChieh Chen. 2018. Inverted Residuals and Linear Bottlenecks: Mobile Networks for Classification, Detection and Segmentation. arXiv preprint arXiv:1801.04381 (2018).

[35] Pierre Sermanet, David Eigen, Xiang Zhang, Michaël Mathieu, Rob Fergus, and Yann LeCun. 2013. Overfeat: Integrated recognition, localization and detection using convolutional networks. arXiv preprint arXiv:1312.6229 (2013).

[36] Abhinav Shrivastava, Rahul Sukthankar, Jitendra Malik, and Abhinav Gupta. 2016. Beyond skip connections: Top-down modulation for object detection. arXiv preprint arXiv:1612.06851 (2016).

[37] Karen Simonyan and Andrew Zisserman. 2015. Very deep convolutional networks for large-scale image recognition. In ICLR.

[38] Bharat Singh, Hengduo Li, Abhishek Sharma, and Larry S Davis. 2018. R-FCN3000 at $30 \mathrm{fps}$ : Decoupling Detection and Classification. In CVPR.

[39] Christian Szegedy, Scott Reed, Dumitru Erhan, Dragomir Anguelov, and Sergey Ioffe. 2014. Scalable, high-quality object detection. arXiv preprint arXiv:1412.1441 (2014).

[40] Jasper RR Uijlings, Koen EA Van De Sande, Theo Gevers, and Arnold WM Smeulders. 2013. Selective search for object recognition. I7CV 104, 2 (2013), 154-171.

[41] Yu Xiang, Wongun Choi, Yuanqing Lin, and Silvio Savarese. 2017. Subcategoryaware convolutional neural networks for object proposals and detection. In WACV. IEEE, 924-933.

[42] Matthew D Zeiler and Rob Fergus. 2014. Visualizing and understanding convolutional networks. In ECCV. Springer, 818-833. 